\title{
The onset of liver glycogen synthesis in fasted-refed lean and genetically obese $(f a / f a)$ rats
}

\author{
G. van de Werve and B.Jeanrenaud \\ Laboratoires de Recherches Métaboliques, Faculty and Department of Medicine, Geneva, Switzerland
}

\begin{abstract}
Summary. Lean and genetically obese ( $f a / f a$ ) rats were fed ad libitum, or fasted for $17 \mathrm{~h}$ and then meal-fed for varying time intervals. During refeeding, glucose-6-phosphatase activity of lean rats declined to the low value that was present in livers of fasted obese rats and which remained unchanged in the obese group during the meal. Refeeding also resulted in increases in hepatic concentrations of glucose-6-phosphate and fructose6-phosphate, fructose 1,6-bisphosphate, fructose-2,6-bisphosphate, $\alpha$-glycerophosphate, pyruvate and lactate in lean and obese rats, absolute values being higher in the fasted obese than in the fasted lean group. Obese animals had higher postprandial portal blood insulin, glucose and lactate concentrations than lean animals. In spite of this, the rate of hepatic glycogen deposition was the same in both groups and was ac-
\end{abstract}

companied by similar glycogen synthase $a$ levels. Following refeeding, phosphorylase was transiently inactivated in livers of lean but not of obese animals, while glycogen synthase was inactivated in both groups. The data suggest that (1) in lean animals refeeding was associated with a stimulation of liver glycolysis, presumably by insulin; (2) in fasted obese rats hepatic glycolysis was already in a stimulated state and was only slightly enhanced further after the meal, in keeping with their unaltered hyperinsulinaemia; (3) there was an increased turnover of liver glycogen or a resistance to insulin stimulation of glycogen synthesis in $f a / f a$ rats during refeeding.

Key words: Liver glycogen metabolism, $f a / f a$ rats, feeding, insulin resistance, glycolysis, fructose-2,6-bisphosphate.
Genetically obese $(f a / f a)$ rats are insulin resistant, a pathology that has been traced to muscle and adipose tissue [1]. In liver as well, insulin resistance was suggested by the observation that the hepatic glucose production in vivo was not suppressed by the high basal hyperinsulinaemia [2] of these obese rodents, the suppression of the process being observed only at pharmacological concentrations $(10 \mathrm{mU} / \mathrm{ml})$ of the hormone [3].

The onset of hepatic glycogen synthesis after a meal is favored by insulin in livers of normal rats [4]. In this tissue $[5,6]$ as in skin [7], glycogen formation was shown not to require an activation of glycogen synthase, but was associated with an inactivation of phosphorylase. Insulin infusion also enhances glycolysis in livers of normal rats clamped at euglycaemia [8].

It was therefore of interest to study the effect of endogenous insulin on liver glycogen formation and glycolytic parameters upon refeeding obese rats, in order to evaluate the nature of their potential hepatic insulin resistance.

\section{Materials and methods}

\section{Animals}

Lean and genetically obese $(f a / f a)$ male rats, weighing about $250 \mathrm{~g}$ and $360 \mathrm{~g}$ respectively, were purchased at the CNRS breeding center (Orléans, France). The animals were kept for one week on standard laboratory chow ( $21 \%$ proteins, 3\% lipids, 39\% carbohydrates, $5 \%$ cellulose, $32 \% \mathrm{H}_{2} \mathrm{O}$, amino acids and minerals, Lacta, Cossonay, Switzerland) prior to use. The rats were then divided into two groups: one was fasted for $17 \mathrm{~h}$ (from 17.00 to 10.00 hours), then given access to food distributed at the bottom of the cages and used 2,6 or $10 \mathrm{~h}$ later (refed); the other group had free access to food (fed ad libitum) and was used at 10,00 hours. Each group of animals was anaesthetized with pentobarbital $(0.1 \mathrm{mg} / \mathrm{g}$ body wt intraperitoneally) at the corresponding experimental feeding time, the abdomen was incised to expose the liver and a loose ligature was placed around the portal vein. Fifteen min later, the liver was excised and rapidly $(1 \mathrm{~s})$ frozen between aluminium blocks cooled in liquid nitrogen. Simultaneously, the ligature was tied and blood samples taken from the vein. Frozen livers were stored at $-80^{\circ} \mathrm{C}$ pending enzyme and metabolite determinations.

\section{Analytical methods}

Plasma glucose [9] and insulin [10] were determined as previously described. Fluorimetric assays of metabolites were performed in depro- 


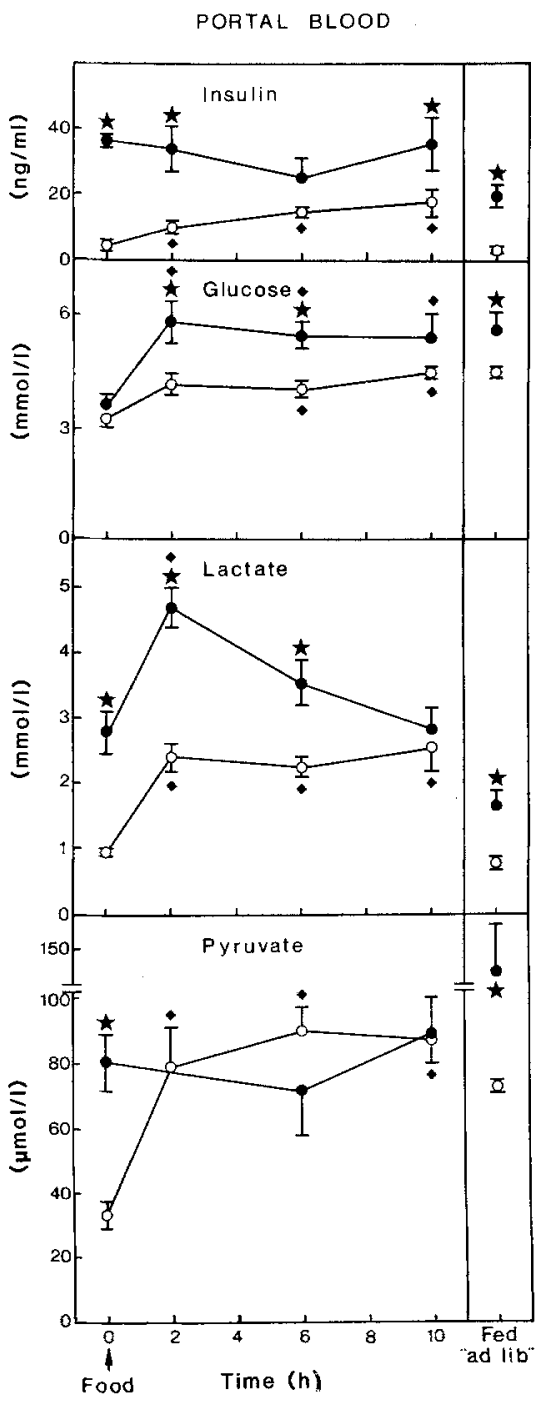

Fig. 1. Time course of feeding on portal vein levels of insulin, glucose, lactate and pyruvate. Blood was taken from lean $(0)$ and obese (O) animals at the indicated times following $17 \mathrm{~h}$ fasting, as described in the Methods section. Time 0 refers to fasted rats prior to onset of refeeding. Fed ad libitum (ad lib) refers to rats which had free access to food throughout the experiment and prior to it. Values are means \pm SEM for 4-6 animals. Statistical significance (unpaired Student's test) of differences are shown between the lean and obese groups $(*: p<$ $0.05)$ and between fasted and fed animals in each group $(\bullet: p<0.05)$

teinized plasma (lactate, pyruvate) and in neutralized trichloroacetic liver extracts (uridine diphosphoglucose, glucose- and fructose6-phosphate, fructose-1,6-bisphosphate, trioses-phosphate, $\alpha$-glycerophosphate, phosphoenolpyruvate, pyruvate and lactate) by standard procedures [11]. Cyclic AMP was assayed in the same extracts [12] purified as described elsewhere [13]. Fructose-2,6-bisphosphate was measured in alkaline liver extracts, after deduction of acid-labile activity, as previously recommended [14]. The liver glycogen content as well as the activities of glycogen synthase and phosphorylase were measured as described elsewhere [6]. Glucose-6-phosphatase activity was assayed at $30{ }^{\circ} \mathrm{C}$ in the presence of $0.2 \%$ deoxycholate as detailed by others [15]. One unit of phosphorylase, synthase or glucose-6-phosphatase was defined as the amount of enzyme converting $1 \mu \mathrm{mol}$ of substrate per min under the conditions of the assay.

\section{Statistical analysis}

Statistical analysis was performed using Student's t-test for unpaired samples. A $p$ value of $<0.05$ was considered statistically significant.

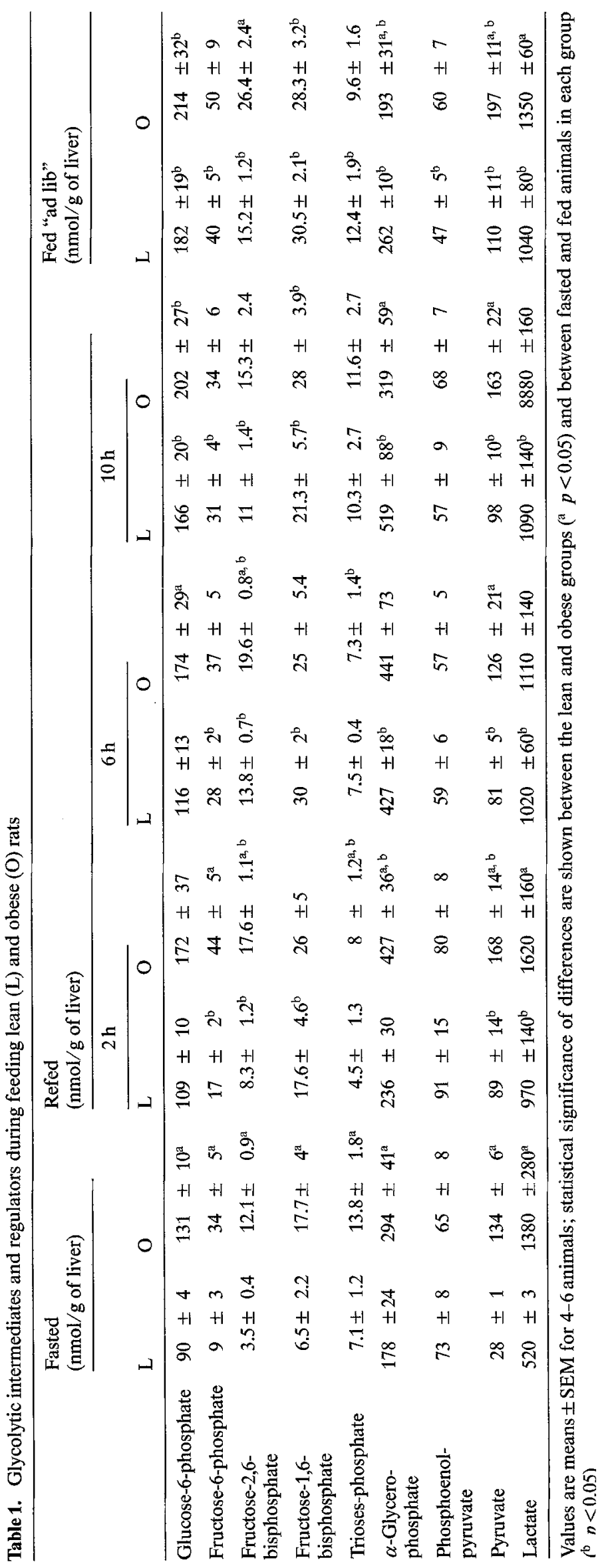



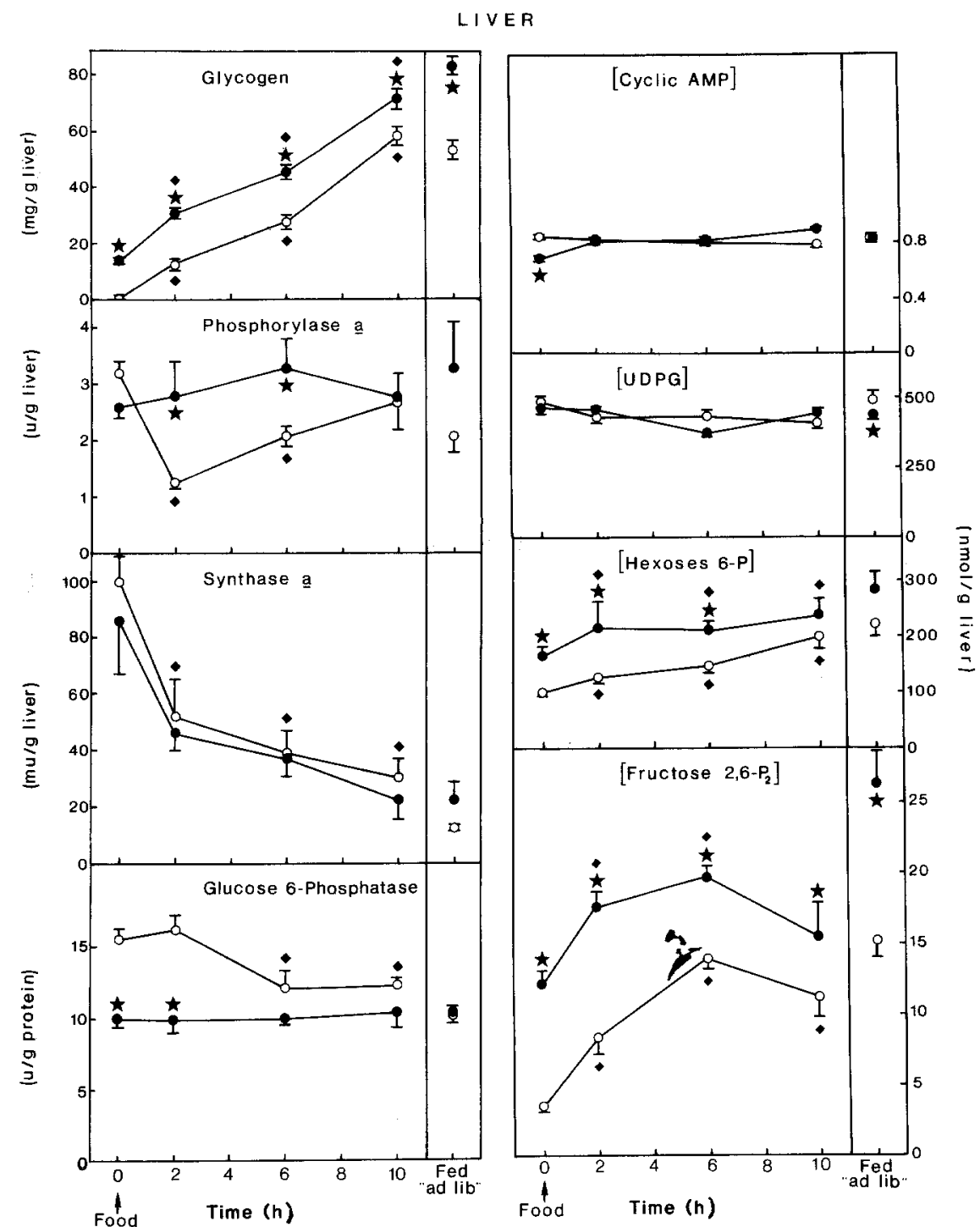

Fig. 2. Left panel: time course of refeeding effect (see Fig. 1) on liver glycogen content, on the activities of phosphorylase $a$, synthase $a$ and glucose-6-phosphatase. Right panel: time course of refeeding effect on the concentrations of cyclic AMP, UDPG, hexoses-6-phosphate and fructose-2,6-bisphosphate. Livers were sampled from lean $(O)$ and obese $(\odot)$ animals at the indicated times as described in the Methods section. Other conditions (feeding time and statistical analysis) are as in Fig. 1

\section{Results}

\section{Changes in portal vein parameters during refeeding}

In the obese rats, fasting levels (time zero) of blood insulin, lactate and pyruvate were markedly elevated compared to controls (Fig.1). Upon refeeding insulin and pyruvate remained at the same high levels in the obese group, while glucose was increased by $50 \%$ throughout the meal and lactate increased transiently. Obese rats fed ad libitum had higher portal vein levels of insulin, glucose, lactate and pyruvate than fed controls. Refeeding lean rats resulted in increased portal blood insulin, glucose, lactate and pyruvate concentrations which reached values that were similar to those of obese at the end of the meal ( 10.00 hours), except for insulin, which always remained lower compared to the obese group.

The elevated lactate and pyruvate levels throughout the meal in the obese rat portal vein as well as the increase in these parameters upon feeding lean rats is con- sistent with changes in hepatic glycolysis (Fig.3, Table 1). Increased lactate production by the liver has been observed in dogs fed a mixed meal [38]. Blood glucose levels were the same in both fasted lean and obese animals. The increase in glycaemia following the meal was more pronounced in the obese group, confirming an earlier report of oral glucose intolerance in $f a / f a$ rats [16] which was attributed to enhanced hepatic glucose production [17].

\section{Hepatic glycogen metabolism during refeeding}

Figure 2 (left panel) shows the glycogen content, the activities of phosphorylase $a$, synthase $a$ and glucose6-phosphatase in livers of lean and obese rats, fasted, refed or ad libitum fed. Under all nutritional conditions hepatic glycogen levels were higher in the obese group, although the rate of glycogen formation was comparable with that measured in livers of lean animals (lean: $7.1 \pm 1$ and obese: $7.4 \pm 0.9 \mathrm{mg}$ glycogen $/ \mathrm{g}$ liver $\mathrm{h}$, mean values \pm SEM calculated from six different feeding time 


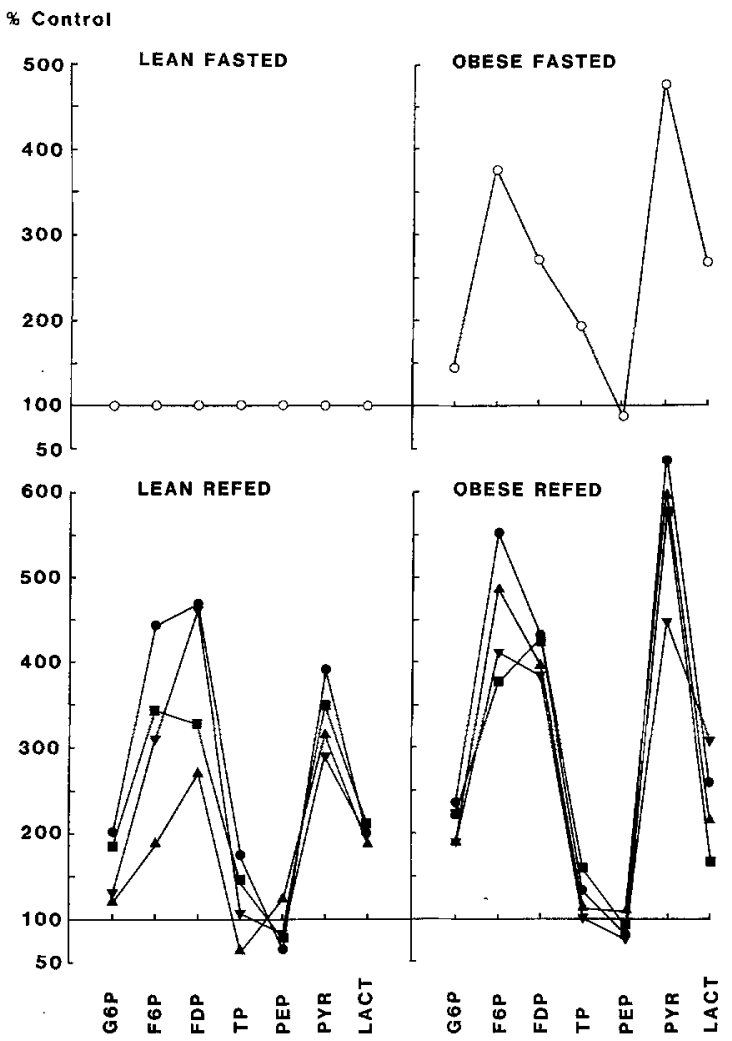

Fig.3. Effect of feeding on glycolytic intermediates. Livers were sampled from lean (left panel) and obese (right panel) rats fasted for $17 \mathrm{~h}$

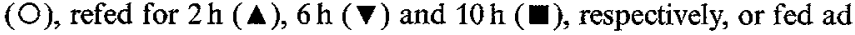
libitum (-). Glycolytic intermediates were assayed as described in the Methods section. The results are expressed as a percentage of the values $(100 \%)$ found in livers of fasted lean rats. Absolute values and statistical analysis can be found in Table 1

intervals). A transient but marked inactivation of phosphorylase was observed in livers of lean rats during refeeding but not in those of refed obese animals. Synthase $a$ levels were identical in the two groups of rats and declined during feeding. In obese rats glucose6-phosphatase activity was unaffected by refeeding. In fasted lean animals glucose-6-phosphatase activity was higher than that of obese and declined during feeding to reach the lower activity found in the obese.

Figure 2 (right panel) shows that in both groups of animals hepatic glycogen synthesis during refeeding was not accompanied by significant changes in UDPG concentrations, but by an increase in the concentration of hexoses-6-phosphate (glucose-6-phosphate and fructose-6-phosphate), and, probably as a consequence, of fructose-2,6-bisphosphate. In lean rats, cyclic AMP levels were unaffected by feeding. In fasted obese rat livers, the concentration of cyclic AMP was lower than in the lean ones, but increased from two hours of refeeding to reach the higher levels found in lean rats.

\section{Hepatic glycolysis during refeeding}

Glycolysis is known to proceed in livers of fasted $f a / f a$ $[18,19]$ but not of fasted lean rats. It was thus of interest to measure changes in glycolytic intermediates during the refeeding of the fasted animals. The results are presented in absolute values in Table 1 . Increased glycolysis is inferred from dynamic changes in metabolic intermediates and illustrated as cross-over plots (Fig. 3). It is apparent that, in lean rats (Fig.3, left panel), refeeding was associated with a stimulation of glycolysis at the level of phosphofructokinase 1 (PFK 1) and pyruvate kinase. Indeed, one can observe a marked increase in the concentration of the substrate of PFK 1 (fructose6-phosphate in equilibrium with glucose-6-phosphate) as well as in that of its product (fructose-1,6-bisphosphate). Such changes are consistent with a stimulation of PFK 1 by fructose-2,6-bisphosphate, which increases upon feeding as described above (see Fig. 2). There was, however, no crossover at the PFK 1 step, probably because of the increase in hexoses-6-phosphate concentration (due to substrate provision by the meal). A profile similar to that of Figure 3 has been observed in normal rats infused with insulin at euglycaemia [8], but with a cross-over at PFK 1 because, in these conditions, glucose-6-phosphate concentrations decreased. The activation of pyruvate kinase in refed lean rats is suggested by the marked accumulation of its product (pyruvate) and slight decrease in its substrate (phosphoenolpyruvate) concentration. The increase in fructose1,6-bisphosphate, a potent stimulator of pyruvate kinase, might have further enhanced this glycolytic step [20]. In addition and as shown by Table 1, a marked accumulation of $\alpha$-glycerophosphate was observed during the refeeding of normal rats, an accumulation that could derive from trioses-phosphate formed by the stimulation of the glycolytic pathway. As further shown in Figure 3 (right panel), livers from fasted obese rats had a glycolytic profile that was comparable to that of livers of the refed lean animals, thus indicating that even in the fasted state the glycolytic pathway was active in the obese group. It is also evident that the same glycolytic steps (PFK 1 and pyruvate kinase) were also further stimulated, albeit slightly, upon refeeding the obese animals.

\section{Discussion}

\section{Glycogen synthesis in the lean rat}

In previous studies we have shown that synthase activation was not a prerequisite for hepatic glycogen synthesis during a meal $[5,6]$. This feature is confirmed in the present work. Actually, there is even an inactivation of the synthase, a finding that has been previously reported $[6,23]$ and attributed to the inhibition of synthase phosphatase by glycogen [24]. Such a negative feedback of glycogen [25-27] on its own synthesis might be useful in preventing excessive accumulation of the polysaccharide [28]. Our results suggest that glycogen synthesis is induced by a stimulation of synthase $a$ via substrate 
availability and/or by positive allosteric modifiers of the enzyme [29], rather than by changes in the synthase $a$ levels.

\section{Glycogen synthesis in the obese rat}

One should expect that the presence of high portal blood insulin concentrations in the obese animals would stimulate liver synthase $a$ activity [29] and enhance glycogen synthesis as soon as meal-derived substrates are available. This was, however, not observed, since the rate of glycogen formation during refeeding was identical in livers of lean and obese rats (Fig. 2). Net glycogen accumulation is the result of the balance between its synthesis (by synthase $a$ ) and its degradation (by phosphorylase $a$ ). Phosphorylase $a$ levels remained elevated throughout the experiment in livers of obese animals. This indicates either that phosphorylase inactivation does not contribute to the net formation of glycogen or that feeding is associated, in livers of obese rats, with an increased rate of glycogen turnover. If the latter possibility were correct, one would conclude that the actual rate of glycogen synthesis was increased in obese rats.

An alternative view for the lack of stimulation of glycogen formation by insulin and of inactivation of phosphorylase in refed obese rats would be that these events are impaired due to a hepatic insulin resistance. Indeed, net liver glucose production is not suppressed in vivo and at euglycaemia in obese animals, despite their high basal blood insulin concentration, while it is completely inhibited in normal controls when insulin is raised to the same value as that prevailing in the obese [3]. The cause for lack of inactivation of liver phosphorylase in refed obese rats is unknown. Amongst several possibilities one may mention: (1) an impaired glucosestimulation of phosphorylase phosphatase, (2) a maintenance of active phosphorylase kinase by high intracellular free calcium and (3) a dysregulation of insulin counter-regulatory hormones. Two of these defects occur in another strain of genetically obese animals, the $o b / o b$ mice: (1) glucose resistance has been reported $[30,31]$ and (2) support for the hypothesis of abnormal calcium handling has been provided by studies on liver mitochondrial calcium transport [21] and in hepatocytes of $o b / o b$ mice [22].

\section{Glycolysis}

Under our experimental conditions, liver glycolysis was found to be stimulated during refeeding both lean and obese animals. The fact that glycolytic parameters were enhanced in the liver of obese rats even when fasted indicate that this pathway is stimulated in these animals, presumably by their prevailing hyperinsulinaemia, and therefore does not become insulin-resistant. These changes in liver glycolysis are consistent with those of portal blood lactate (Fig. 1).
It has to be underscored that a crossover plot (Fig. 3) does not give information on the glycolytic flux. With this limitation in mind, one may question a contribution of gluconeogenesis to glycogen synthesis $[32,33]$ unless a metabolic zonation of these opposite pathways exists in the liver. Such a zonation has been previously proposed [34]. The present experimental approach is unable to discriminate between these potential cell subpopulations. One should note that recent reports suggest that the origin of the gluconeogenic precursors for glycogen synthesis might lie within the liver itself [35-38], and that these precursors might be produced by insulin-stimulated hepatic glycolysis [39]. In this respect, it is interesting to recall that concurrent gluconeogenesis and glycolysis have been observed in primary cultures of hepatocytes $[40,41]$.

In summary, our results indicate that: (a) glycolysis in liver of genetically obese rats is continuously overstimulated by the high basal insulinaemia and, due to this, only slightly stimulated further upon feeding; (b) elevated phosphorylase $a$ levels of livers of obese rats might be an appropriate metabolic adapation that would attempt coping with an enhanced glycogen synthesis due to hyperinsulinaemia. An enhanced glycogenolysis resulting from elevated phosphorylase $a$ levels might be the consequence of hepatic insulin resistance or of a dysregulation of at least some of the insulin counter-regulatory hormones. In the latter hypothesis the apparent insulin resistance could be extrahepatic in its nature.

\section{References}

1. Jeanrenaud B (1985) An hypothesis on the aetiology of obesity: dysfunction of the central nervous system as a primary cause. Diabetologia $28: 502-513$

2. Terrettaz J, Jeanrenaud B (1983) In vivo hepatic and peripheral insulin resistance in genetically obese $(f a / f a)$ rats. Endocrinology 112: $1346-1351$

3. Terrettaz J, Assimacopoulos-Jeannet F, Jeanrenaud B (1986) Severe hepatic and peripheral insulin resistance as evidenced by euglycemic clamps in genetically obese $f a / f a$ rats. Endocrinology 118: $674-678$

4. Stalmans W, van de Werve G (1981) Regulation of glycogen metabolism by insulin. In: Hue L, van de Werve G (eds) Short-term regulation of liver metabolism. Elsevier/North-Holland, Amsterdam, pp 119-138

5. van de Werve G, Sestoft L, Folke M, Kristensen LØ (1984) The onset of liver glycogen synthesis in fasted-refed rats. Effects of streptozotocin diabetes and of peripheral insulin replacement. Diabetes 33: 944-949

6. van de Werve G, Jeanrenaud B (1984) Synthase activation is not a prerequisite for glycogen synthesis in the starved liver. Am J Physiol 247: E271-E275

7. Harmon CS, Phizackerley PJA (1983) The effects of starvation and refeeding on glycogen metabolism in tail skin. Biochem $\mathrm{J} 212$ : 679-683

8. Terrettaz J, Assimacopoulos-Jeannet F, Jeanrenaud B (1986) Inhibition of hepatic glucose production by insulin in vivo in the rat: contribution of glycolysis. Am J Physiol 250: E346-E351

9. Huggett ASG, Nixon DA (1957) Enzymic determination of blood glucose. Biochem J 66: 12P 
10. Herbert V, Lau KS, Gottlieb CW, Bleicher SJ (1965) Coated charcoal immunoassay of insulin. J Clin Endocrinol Metab 25: 1375-1384

11. Bergmeyer HU (ed) (1974) Methods of enzymatic analysis. Verlag Chemie, Weinheim and Academic Press, New York, pp 184-185

12. Gilman AG (1970) A protein binding assay for adenosine $3^{\prime}: 5^{\prime}$ cyclic monophosphate. Proc Natl Acad Sci USA 67: 305-312

13. van de Werve $G$, van den Berghe $G$, Hers $H G$ (1974) A simplified procedure for the assay of adenosine $3^{\prime}, 5^{\prime}$-monophosphate by the activation of liver phosphorylase. Eur J Biochem 41: 97-102

14. van Schaftingen E, Lederer B, Bartrons R, Hers HG (1982) A kinetic study of pyrophosphate: fructose-6-phosphate phosphotransferase from potato tubers. Application to a microassay of fructose 2,6-bisphosphate. Eur J Biochem 129: 191-195

15. Kitcher SA, Siddle K, Luzio JP (1978) A method for the determination of glucose-6-phosphatase activity in rat liver with $\left[\mathrm{U}-{ }^{14} \mathrm{C}\right]$ glucose-6-phosphate as substrate. Anal Biochem 88: 29-36

16. Ionescu E, Sauter JF, Jeanrenaud B (1985) Abnormal oral glucose tolerance in genetically obese $(f a / f a)$ rats. Am J Physiol 248: E500-E506

17. Rohner-Jeanrenaud F, Proietto J, Ionescu E, Jeanrenaud B (1986) Mechanisms of abnormal oral glucose tolerance of genetically obese $\mathrm{fa} / \mathrm{fa}$ rats. Diabetes 35: 1350-1355

18. McCune SA, Durant PJ, Jenkins PA, Harris RA (1981) Comparative studies on fatty acid synthesis, glycogen metabolism, and gluconeogenesis by hepatocytes isolated from lean and obese Zucker rats. Metabolism 30: 1170-1178

19. Hue L, van de Werve G, Jeanrenaud B (1983) Fructose 2,6-bisphosphate in livers of genetically obese rats. Biochem $J$ 214: 1019-1022

20. Rognstad R (1982) Control of lactate gluconeogenesis by glucose in rat hepatocytes. Arch Biochem Biophys 217:498-502

21. Fraser DR, Trayhurn P (1983) Mitochondrial $\mathrm{Ca}^{2+}$ transport in lean and genetically obese $(o b / o b)$ mice. Biochem J 214: 163-170

22. van de Werve G, Assimacopoulos-Jeannet F, Jeanrenaud B (1983) Altered liver glycogen metabolism in fed genetically obese mice. Biochem J 216: 273-280

23. Larner J (1972) Insulin and glycogen synthase. Diabetes 21: $428-438$

24. De Wulf H, Stalmans W, Hers HG (1970) The effect of glucose and of a treatment by glucocorticoids on the activation in vitro of liver glycogen synthetase. Eur J Biochem 15:1-8

25. Fleig WE, Nöther-Fleig G, Steudter S, Enderle D, Ditschuneit H (1986) Effect of down-regulation and return of insulin receptors on glycogen synthesis in cultured rat hepatocytes. Biochem Biophys Acta 888: 191-198

26. Watts C, Malthus RS (1980) Liver glycogen synthase in rats with a glycogen-storage disorder. The role of glycogen in the regulation of glycogen synthase. Eur J Biochem 108: 73-77

27. Watts C, Redshaw JR, Gain KR (1982) The activation of glycogen synthase in hepatocytes from rats with a glycogen storage disorder (gsd/gsd). Febs Lett 144: 231-234
28. van de Werve G (1981) Non-hormonal control of glycogenolysis. In: Hue L, van de Werve G (eds) Short-term regulation of liver metabolism. Elsevier/North-Holland, Amsterdam, pp 93-104

29. Ciudad CJ, Bosch F, Guinovart JJ (1981) Insulin activation of basal hepatic glycogen synthase. Febs Lett 129: 123-126

30. Kreutner W, Springer SC, Sherwood JE (1975) Resistance of gluconeogenic and glycogenic pathways in obese-hyperglycemic mice. Am J Physiol 228: 663-671

31. Smith SA, Cawthorne MA, Levy AL, Simson DL (1983) Defective activation by glucose of hepatic glycogen synthesis in the obese hyperglycaemic mouse. Biochem J 216: 491-494

32. Foster DW (1984) From glycogen to ketones - and back. Diabetes 33: 1188-1199

33. Katz J, McGarry JD (1984) The glucose paradox: is glucose a substrate for liver metabolism? J Clin Invest 74: 1901-1909

34. Jungermann K, Katz N (1982) Functional hepatocellular heterogeneity. Hepatology 2: 385-395

35. Bergman RN, Beir JR, Hourigan PM (1982) Intraportal glucose infusion matched to oral glucose absorption. Lack of evidence for "gut factor" involvement in glucose storage. Diabetes 31:27-35

36. Bratusch-Marrain PR, Waldhäusl WK, GasizS, Korn A, Nowotny P (1980) Oral glucose tolerance test: effect of different glucose loads on splanchnic carbohydrate and substrate metabolism in healthy man. Metabolism 29:289-295

37. Radziuk J, Inculet R (1983) The effects of ingested and intravenous glucose on forearm uptake of glucose and glucogenic substrate in normal man. Diabetes 32:977-981

38. Davis MA, Williams PE, Cherrington AD (1984) Effect of a mixed meal on hepatic lactate and gluconeogenic precursor metabolism in dogs. Am J Physiol 247: E362-E369

39. Soley M, Chieri R, Herrera E (1985) Short-term insulin infused through the portal vein enhances liver gluconeogenesis and glycogenesis from $\left[3-{ }^{14} \mathrm{C}\right]$ pyruvate in the starved rat. Int $\mathbf{J}$ Biochem 17: 689-693

40. Amatruda JM, Salmanick AI, Chang CL (1985) Hormone effects on glycogen accumulation in rat hepatocytes. Proceedings of the 67th meeting of the Endocrine Society, Abstract No 369, p93

41. Spence JT, Koudelka AP (1985) Pathway of glycogen synthesis from glucose in hepatocytes maintained in primary culture. J Biol Chem 260: 1521-1526

Received: 13 August 1986

and in revised form: 20 January 1987

Dr. G.van de Werve

Department of Nutrition

Faculty of Medicine

University of Montreal

2405, Chemin de la Cote-Ste-Catherine

H3T 1A8 Montreal, Canada 